\title{
Circuit theory approach to stability and passivity analysis of nonlinear dynamical systems
}

\author{
Muzaffer Ates ${ }^{1}$ \\ ${ }^{1}$ University
}

November 25, 2020

\begin{abstract}
In this paper, we address the problem of global asymptotic stability and strong passivity analysis of nonlinear and nonautonomous systems controlled by second-order vector differential equations. First, we construct this system or the differential equation from a nonlinear time varying network of the circuit theory. Our system and with its real energy function generalize and improve upon some well-known studies in the literature. This system and its special forms have ample applications in many scientific investigations. We realized that most of the first- and second-order ordinary differential equations can be represented by LRC circuits. So, the energy (Lyapunov) functions of the systems can be constructed directly without much trial and error. By this way, the application of Lyapunov's direct method may become a standard technique for physical systems. We illuminate this idea with many applications and improvements. We also compare the Lyapunov stability theory with Hamiltonian and Lagrangian systems in the sense of conservative and dissipative systems. Then, we provide new explicit stability and passivity results with minimum criteria.
\end{abstract}

\section{Hosted file}

MMAS.pdf available at https://authorea.com/users/378675/articles/495108-circuit-theoryapproach-to-stability-and-passivity-analysis-of-nonlinear-dynamical-systems 